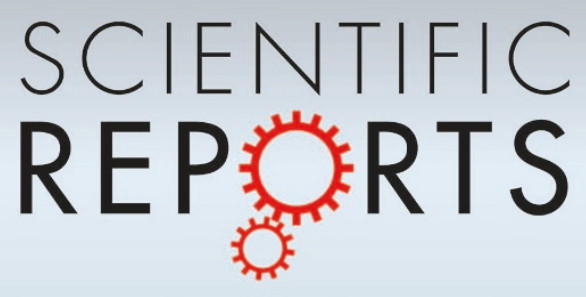

OPEN

SUBJECT AREAS:

OPTICAL TECHNIQUES

STRUCTURE OF SOLIDS AND

LIQUIDS

CHARACTERIZATION AND

ANALYTICAL

TECHNIQUES

Received

7 October 2014

Accepted

4 December 2014

Published

6 January 2015

Correspondence and requests for materials should be addressed to C.D. (christian.david@ psi.ch)

\section{Following the dynamics of matter with} femtosecond precision using the X-ray streaking method

\author{
C. David' ', P. Karvinen' ', M. Sikorski' ', S. Song' ', I. Vartiainen' ', C. J. Milne' , A. Mozzanica', Y. Kayser',

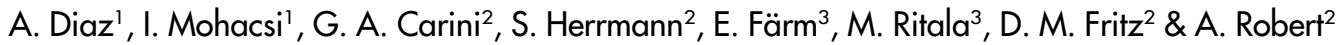

'Paul Scherrer Institut, CH-5232 Villigen, Switzerland, '2SLAC National Accelerator Laboratory, Menlo Park, CA 94025, USA,

${ }^{3}$ Department of Chemistry, University of Helsinki, Helsinki Fl-00014, Finland.

X-ray Free Electron Lasers (FELs) can produce extremely intense and very short pulses, down to below 10 femtoseconds (fs). Among the key applications are ultrafast time-resolved studies of dynamics of matter by observing responses to fast excitation pulses in a pump-probe manner. Detectors with sufficient time resolution for observing these processes are not available. Therefore, such experiments typically measure a sample's full dynamics by repeating multiple pump-probe cycles at different delay times. This conventional method assumes that the sample returns to an identical or very similar state after each cycle. Here we describe a novel approach that can provide a time trace of responses following a single excitation pulse, jitter-free, with fs timing precision. We demonstrate, in an X-ray diffraction experiment, how it can be applied to the investigation of ultrafast irreversible processes.

T he understanding of phenomena in matter often relies on the investigation of its structure, meaning its atomic composition, and its dynamics describing how the structure undergoes changes. Many fundamental dynamic processes, such as the formation or breaking of chemical bonds, are related to the motion of atoms. The time scales of the vibrational movement of atoms typically lie in the sub-picosecond range. The advent of femtosecond lasers has provided a powerful tool for the study of transient matter. In pump-probe experiments they are used to push a system out of equilibrium using a pulsed excitation (pump) and the subsequent relaxation dynamics are measured by applying a second pulse to determine the status of a particular property of the sample after a given delay (probe). With the advent of suitable sources such as Laser Plasmas ${ }^{1,2}$, High-Harmonic Generation $^{3}$, Femto-Slicing ${ }^{4}$, and X-ray Free-Electron Lasers ${ }^{5-7}, \mathrm{X}$-ray probe pulses are receiving increasing attention, as X-rays offer a rich variety of resonantly enhanced contrast mechanisms to reveal element-specific and even chemical information. X-ray based spectroscopic methods applied on a femtosecond time scale ${ }^{8}$ can also directly interrogate local changes of electronic states. When using multi-keV or hard X-rays, the structural dynamics of matter can be probed by diffraction experiments $\mathrm{s}^{1,2,9,10}$.

All these experiments measure one particular delay after the arrival of the pump pulse at a time. Thus, these techniques are essentially limited to the study of reversible processes that reproducibly return to their ground state after each pump-probe cycle, or they require an identical, fresh sample for each shot, with identical orientation in the case of diffraction experiments. This has hindered pump-probe studies of highly excited materials at nearsolid densities as they reach local thermal equilibrium: the so-called "warm dense matter" regime ${ }^{11}$. Since these very high-energy density experiments permanently damage the samples with each shot, and constant supply of fresh identical samples is only possible in some experiments, it is most useful to find a way to get complete time traces of the response from a single X-ray pump pulse.

Another important limitation for ultrafast time-resolved experiments at X-ray FEL sources has been the loss in time resolution due to the timing jitter between the pump and probe pulses. Typically, the external pump lasers are synchronized to the X-ray FEL via radiofrequency phase-locking, resulting in short term jitter of more than $100 \mathrm{fs}^{12}$, and long term drifts in the ps range. The development of more advanced techniques, e.g. exploiting the terahertz emission from the FEL undulator ${ }^{13}$ or the signal from electron bunch monitors ${ }^{14}$ for the stabilization of the X-ray and optical pulses, is a topic of intense research. A pragmatic and robust method involves monitoring the relative time delay on a per-shot basis and re-sorting the data accordingly. This approach can reduce timing errors to below $10 \mathrm{fs}^{15}$, but it obviously requires data sets collected using many pump pulses and is therefore of 
limited use for single-shot experiments. A fundamental way to avoid timing jitter between pulses is to split them from the same parent pulse and to control their temporal separation by a delay line. Several such instruments based on mirrors ${ }^{16,17}$ and Bragg crystals ${ }^{18,19}$ have been developed for soft and hard X-ray FEL radiation, respectively. However, both approaches only provide one delay time per X-ray pulse.

We pursue a split-and-delay approach based on diffraction gratings as shown in Figure 1. A set of beam splitter gratings $S_{n}$ with different periods $p_{n}$ diffracts a small fraction of the incoming radiation into a fan of beams. A second set of gratings $R_{n}$ is positioned half way between $S_{n}$ and the sample to recombine the diffracted beams with the direct, undiffracted beam at the sample position. For this purpose, the recombiner periods $q_{n}$ must be half the period $p_{n}$ of the corresponding $\mathrm{S}_{\mathrm{n}}$ grating. The deflection $\Delta x_{n}=a \lambda / 2 p_{n}$ in the recombiner plane with respect to the undiffracted beam results in a delay $\Delta t_{n}$ of

$$
\Delta t_{n}=\frac{a}{2 c} \frac{\lambda^{2}}{p_{n}^{2}}=\frac{a}{8 c} \frac{\lambda^{2}}{q_{n}^{2}}
$$

where $a$ denotes the distance between $S_{n}$ and the sample, $c$ the speed of light, and $\lambda$ the $\mathrm{X}$-ray wavelength. For weak $\mathrm{S}_{\mathrm{n}}$ gratings - meaning gratings of low diffraction efficiency - most of the intensity remains in the undiffracted beam, which can be used as a pump pulse to excite the sample. This pulse is followed by a series of probe pulses, each a diffracted beam, with delays that are precisely defined by the geometrical parameters and not subject to any pump-probe jitter. The probe beams diverge again downstream of the sample, and a streak of delayed probe pulses can be recorded on a detector array, analogously to commonly used electron streak cameras. No time resolving detector is required.

The positive and negative diffraction orders of each splitter grating $\mathrm{S}_{\mathrm{n}}$ create a symmetric pair of beams propagating towards the recombiner gratings $R_{n}$. In our set-up depicted in Figure 1, the beams diffracted in the upward direction are deflected by the corresponding $R_{n}$ gratings to probe the pumped region of the sample. However, the beams diffracted downwards are deflected by $R_{n}$ gratings having a slightly larger period, in order to hit the sample at a slightly different position than the pump beam and the probe beams (see inset of
Figure 1). The Bragg reflection of these beams can be used to provide the unpumped response for the very same shot as a reference.

In addition to the above-mentioned intrinsic absence of jitter, the availability of several probe beams, and the possibility to record the unpumped response, the experimental setup described here has several particular properties, that make it robust and easy to use at X-ray FEL sources:

- As the $\mathrm{R}_{\mathrm{n}}$ gratings are placed half-way between the $\mathrm{S}_{\mathrm{n}}$ gratings and the sample, the set-up is achromatic in the sense that it can accept the full range of photon energies of the self-amplified spontaneous emission (SASE) without losing the intersection point of the pump and probe beams.

- Contrary to set-ups based on reflective optics, the use of transmission gratings makes the set-up insensitive to mechanical drift and vibrations. The deflection angles of the beams are determined by the grating periods, they are not affected by lateral displacements of the gratings, and are very tolerant with respect to changes of the incidence angle onto the gratings.

- Due to the use of two gratings in each delayed beam, the pulse fronts remain parallel to that of the pump beam. This avoids the pulse stretching effect often encountered when using diffractive optics $^{20}$.

We implemented such an experiment at the XCS instrument of the Linac Coherent Light Source (LCLS) ${ }^{21}$, operated at $4.5 \mathrm{keV}$ photon energy and $40 \mathrm{fs}$ pulse length. The essential design parameters are listed in Table 1. The set-up comprises each 15 delayed probe and reference channels with delays spanning over more than a picosecond. As can be seen from eq. 1 , the time delays scale linearly with the length of the setup $a$, and inverse to the square of the grating periods. In order to achieve delays of up to 1.277 ps at $4.5 \mathrm{keV}$ photon energy, we chose $a$ to be $12.2 \mathrm{~m}$, limited by the dimensions of the experimental hutch. In spite of this large distance, the required periods $q_{n}$ need to be as small as $17.4 \mathrm{~nm}$, which is close to the fabrication limits of nanolithographic techniques. Moreover, the grating periods need to be exact within extremely narrow tolerances. Furthermore, due to their nanoscale dimensions, the gratings for long delays could only be made with shallow line profiles, making the gratings and thus the corresponding channels very inefficient, see Table 1 . More information

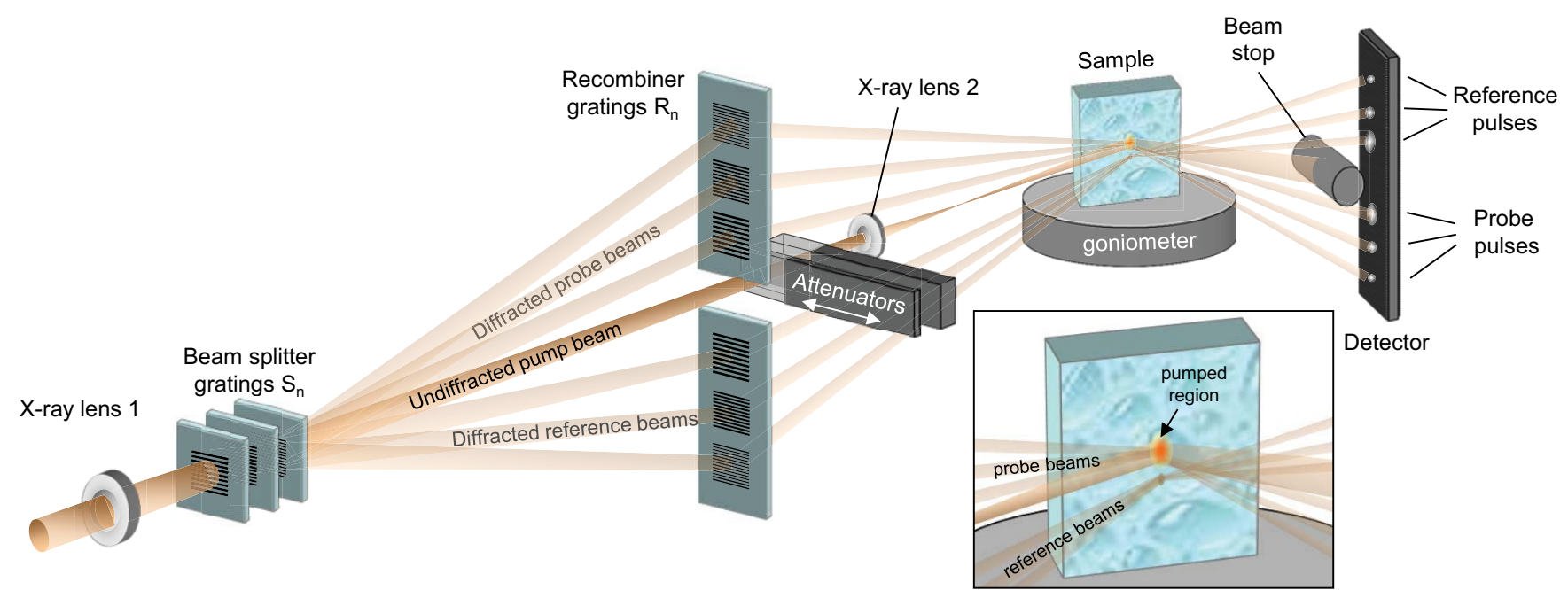

Figure $1 \mid \mathrm{X}$-ray streaking principle. Diffraction gratings are used to create a multiple split-and-delay line. The upstream X-ray lens 1 focuses the XFEL beam onto the sample. The undiffracted (direct) beam serves as a pump, and can be attenuated and focused independently by X-ray lens 2 . The beams diffracted upwards by the splitter gratings $S_{n}$ are redirected towards the sample by the recombiner gratings $R_{n}$, and probe the pumped sample region with defined delays. The beams diffracted downwards by $S_{n}$ are steered to a region of the sample that is $100 \mu \mathrm{m}$ below the pump beam (see inset) to provide reference signals of the unpumped response on the very same shot. All beams are recorded separately on a detector array. Only three delayed beam pairs are shown for simplicity. The sample's scattering plane is chosen perpendicular to that of the gratings, in order to minimize coupling of the scattering angles. 


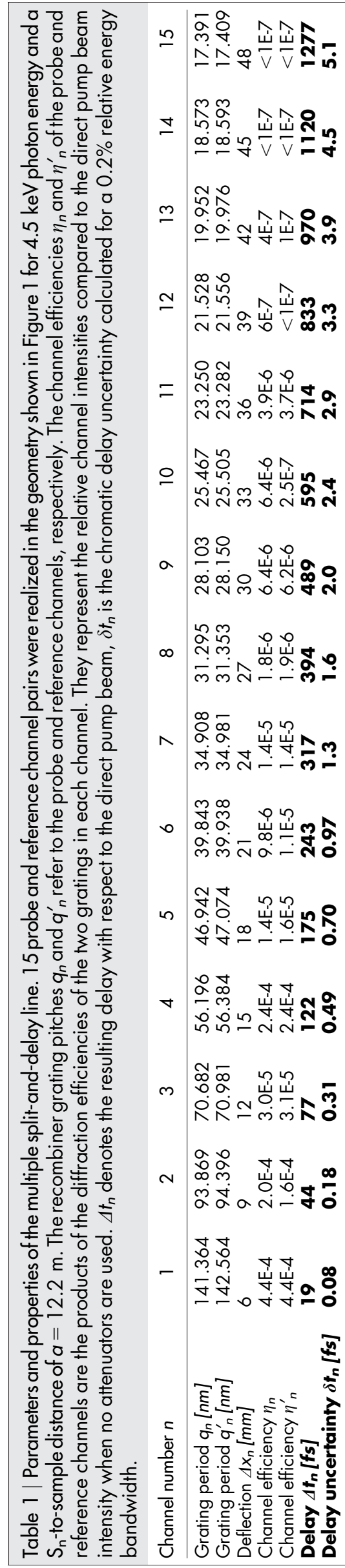

on the grating fabrication tolerances and a possible route to improve the channel efficiency is given in the methods section. It should be noted at this point that the maximum delay demonstrated in the described experiment does not represent a fundamental limit. As the delay scales proportionally with the sqaure of the X-ray wavelength (see eq. 1), a delay range of more than 11 ps could be covered at $1.5 \mathrm{keV}$ photon energy using the same grating periods $p_{n}$ and $q_{n}$ and the same $\mathrm{S}_{\mathrm{n}}$-to-sample length $a$.

The multiple split-and-delay line was used in the scattering geometry shown in Figure 1 to record the Bragg reflection from a Bismuth $<111\rangle$ crystal. We chose a scattering geometry in the horizontal plane in order to ensure that the delayed beams, which are incident at varying vertical angles, all fulfilled the Bragg condition. Figure 2 displays averaged and single shot data of the Bragg-reflected intensities in the case where the direct pump beam was blocked using the attenuator near the $R_{n}$ plane. Although the signal level drops rapidly with increasing delay, even resulting in several "dead" channels, we can clearly observe the delayed streaks of probe and reference pulses. In particular, it should be noted that the $15^{\text {th }}$ channel can still be disinguished from the detector noise level even for the single shot recordings.

The time delays depend on the grating periods and grating distances, both of which can be determined with a relative accuracy of better than $10^{-3}$. The dominating uncertainty of the delays is given by the fact that, according to eq. 1, the path length and therefore the delay is wavelength dependent. At LCLS the beam is subject to a $0.5 \%$ shot-to-shot wavelength jitter related to fluctuations of the accelerator energy ${ }^{4}$. As the latter is monitored for each shot, its effect on the delay is known. The relative spectral width of the individual pulses is limited by the SASE process to $\delta \lambda / \lambda \approx 0.2 \%{ }^{6}$, resulting in $\delta t / \Delta t=2 \delta \lambda / \lambda=0.4 \%$. The timing uncertainty $\delta t_{n}$ is only a few fs even for the longest delays (see Table 1), which is well below the $\mathrm{X}$-ray FEL pulse length itself.

The motivation for using $\mathrm{Bi}<111>$ as the sample in these diffraction experiments was to investigate whether phonon oscillations could be observed. Their effect on the Bragg reflectivity of Bismuth has been studied in a number of experiments using infra-red pump lasers for excitation ${ }^{1,8,9}$. We collected the X-ray streaking signal for several thousand pump events at various pump levels between $1 \times 10^{9} \mathrm{~W} / \mathrm{cm}^{2}$ and just below the damage threshold which was found to be at $2 \times 10^{12} \mathrm{~W} / \mathrm{cm}^{2}$. We tried a variety of experimental settings regarding the pump and probe spot sizes and found no evidence of any oscillatory behaviour in the probe streak. It is unclear whether the contrast of the phonon oscillation signal was too low to be distinguished from shot-to-shot fluctuations of the measured signals or whether no phonon oscillations can be excited with multi$\mathrm{keV} \mathrm{X}$-rays at pump fluences below the damage threshold. The latter explanation is plausible in view of the fact that IR-pumped measurements on phonon oscillations in Bi typically require pump intensities within a factor of two from the stability limit ${ }^{1,9}$.

It is expected that the Bragg reflectivity decreases rapidly once the crystal lattice of the sample disorders for pump levels above the damage threshold. We can follow the dynamics of this effect as displayed in Figure 3. In the unpumped case one can observe the same probe and reference streak as in Figure 2, though recorded using a 2dimensional pixel detector. The three single-shot measurements were performed at a pump fluence far beyond the damage threshold. The probe streak measurement dies out with a decay time of about 50-70 fs, which is somewhat longer than the nominal pump pulse length of $40 \mathrm{fs}$. The order of the atomic lattice obviously vanishes within a few tens of fs, which is consistent with observations by ultrafast electron diffraction ${ }^{22}$. The fact that the reference streak signal remains constant proves that the change in Bragg reflectivity is limited to the pumped region. A more precise observation of the lattice dynamics would require a systematic variation of the pump fluence, which was not possible during the available beam time. 


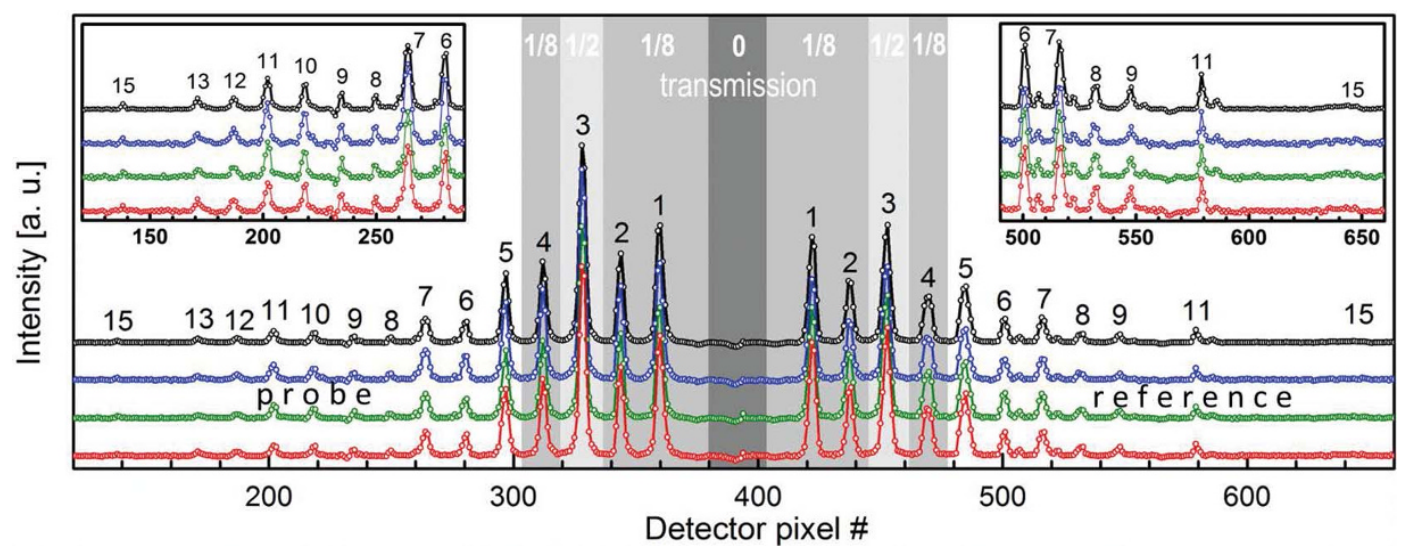

Figure $2 \mid$ X-ray diffraction data of 15 pairs of delayed probe and reference pulses. The data were taken with the set-up sketched in Fig. 1 using a Bi $<111>$ crystal as sample. The black curve shows the average over twelve XFEL shots, while the coloured curves are single shot data. The curves are shown on a linear scale and are offset in the vertical direction for clarity. The grey regions mark the channels that were reduced in transmission by additional attenuators (not shown in Fig. 1) on the $\mathrm{R}_{\mathrm{n}}$ gratings in order to make better use of the dynamic range of the strip detector. The direct pump beam was completely blocked. The insets show probe and reference signals for long delays ( $n \geq 6, \Delta t \geq 243 \mathrm{fs}$ ).

These very first X-ray streaking measurements are unique, as they directly show the evolution of the femtosecond response of a sample following a single, destructive pump pulse, free of timing jitter. The presented experimental set-up can be further improved in particular with respect to the channel efficiency and shot-to-shot fluctuations, as described in the methods section.

A variety of novel experiments could be performed by X-ray streaking. One could, for example, use protein crystals as a sample to directly determine the required pulse length in serial nano-crystallography at $\mathrm{X}$-ray FELs ${ }^{23}$. The damage mechanisms and time scales are of high relevance in structural biology and have so far only been investigated by calculations and indirect measurements ${ }^{24}$. Changes in the X-ray absorption on the femtosecond scale could be observed using the multiple split-and-delay line by placing sample and detector in a transmission geometry. Such measurements could reveal the dynamics of processes following multiple core-shell ionization processes or the formation and dissociation of chemical bonds. In this context, the method could also be used with an external pump laser or other excitation mechanisms. This addition would greatly increase the variety of accessible phenomena, and even though it means losing intrinsic timing with respect to the excitation event, the relative timing of the response would still be accurate.

In summary, we have developed a novel technique for X-ray pump-probe measurements that can provide a series of probe pulses for each pump event. The probe beams have different delays with respect of the excitation pulse, which are intrinsically free of timing jitter, and accurate to the femtosecond level. The technique also provides reference pulses with the same delays that provide information of the unpumped sample for normalization purposes. In a demonstrator experiment at $4.5 \mathrm{keV}$ photon energy, we have presented single-shot measurements of the Bragg reflectivity of a Bismuth crystal pumped with a fluence level far beyond the damage threshold. We found that the reflectivity decays within a few tens of femtoseconds, indicating a fast loss of order in the crystal structure. This unique type of ultra-fast measurements opens up a new path towards the investigation of matter subject to extreme excitation levels that cannot easily be investigated by conventional pump-probe techniques requiring many repetitions of pump-probe cycles at different delay values.

\section{Methods}

Grating fabrication. All gratings were generated using a $100 \mathrm{keV}$ electron-beam writer (Vistec EBPG5000Plus). As the $\mathrm{S}_{\mathrm{n}}$ gratings were subject to the full LCLS beam, they were made from polished $10 \mu \mathrm{m}$ thick diamond membranes (Diamond Materials $\mathrm{GmbH}$ ) to avoid beam damage. Details on the fabrication of diamond diffractive X-ray optics and the radiation hardness in X-ray FEL beams can be found elsewhere ${ }^{25}$. Each diamond grating had an area of $1 \mathrm{~mm} \times 1 \mathrm{~mm}$ to accept the full LCLS beam. As the $R_{n}$ gratings only received much lower fluence they could be made on silicon nitride membranes. In analogy to a process for the fabrication of diffractive lenses ${ }^{26}$, we used a line doubling procedure based on the coating with Iridium using an Atomic Layer Deposition (ALD) process ${ }^{27}$. The $\mathrm{R}_{\mathrm{n}}$ gratings were $500 \mu \mathrm{m} \times 500 \mu \mathrm{m}$ in size.

The diffraction efficiency of all gratings was measured with synchrotron radiation at the cSAXS station of the Swiss Light Source. The channel efficiencies listed in Table 1 are the efficiency products of both gratings in each channel.

Experimental setup. The experiments were performed at the XCS instrument of LCLS. To reduce absorption losses all components except for the sample and the detectors were placed inside helium-filled enclosures, separated from the beam line vacuum by a $100 \mu \mathrm{m}$ thick diamond window. The attenuation of the setup was $88 \%$ up to the sample and another $40 \%$ between the sample and the detectors placed $1.6 \mathrm{~m}$ further downstream.

We used the full SASE emission from LCLS at $4.5 \mathrm{keV}$ photon energy, $2 \mathrm{~mJ}$ pulse energy and $40 \mathrm{fs}$ pulse length throughout the experiments. The delayed beams were focused onto the sample by a Beryllium refractive lens (see Fig. 1, X-ray lens 1) giving a spot size close to the diffraction limit of $\approx 1 \mu \mathrm{m}$. The size of the direct beam was increased to $\approx 30 \mu \mathrm{m}$ by an additional refractive lens (see Fig. 1, X-ray lens 2 ) to facilitate overlap of the pump and the probe beams. In order to achieve a positioning accuracy of the probe beams with respect to the pump beam of $10 \mu \mathrm{m}$ or better, the grating diffraction angles need to be precise to better than $\approx 1 \mu \mathrm{rad}$, requiring the grating periods to have a relative accuracy of better than $\approx 10^{-4}$. For the finest $R_{n}$ grating this means that its pitch of $q_{n}=17.391 \mathrm{~nm}$ needs to be accurate within $\approx 0.002 \mathrm{~nm}$.

The alignment of the probe beams and the reference beams with respect to the pump beam was performed by placing a high resolution $(\sim 2 \mu \mathrm{m}) \mathrm{X}$-ray camera in the sample position, and tuning the rotation angles of the $S_{n}$ gratings around the optical axis as well as the distances between the gratings and the sample. A spatial overlap of clearly better than $10 \mu \mathrm{m}$ could be obtained.

The Bismuth $<111>$ crystal consisted of a cleaved bulk sample (by Mateck $\mathrm{GmbH}$ ) providing single crystal domains of several $\mathrm{mm}$ in size. The Bragg angle was $20.15^{\circ}$, the mosaic spread of the reflections was typically $0.1 \mathrm{mrad}$, resulting in an accepted bandwidth of about $10 \mathrm{eV}$, which matches the bandwidth of a single SASE pulse $(\delta E / E \approx 0.2 \%, \delta E \approx 9 \mathrm{eV})$. The damage threshold of Bi was found to be at $\approx 2 \times 10^{12} \mathrm{~W} / \mathrm{cm}^{2}$.

Data acquisition and data treatment. For the data shown in Fig. 2, we used a Gotthard strip detector ${ }^{28}$ with $50 \mu \mathrm{m}$ pitch. A series of 10.000 shots was acquired at $120 \mathrm{~Hz}$ repetition rate, while monitoring the accelerator energy and the photon pulse intensity for each shot. We found that the relative peak heights varied substantially from shot to shot, and that this variation is clearly correlated with the accelerator energy and pulse intensity. The data displayed in Fig. 2 were selected to have similar accelerator energies and pulse energies close to the mean values of the series, and therefore exhibit very similar peak heights.

The data shown in Fig. 3 were collected with a CS-PAD $140 \mathrm{k}$ detector module ${ }^{29}$ with $110 \mu \mathrm{m} \times 110 \mu \mathrm{m}$ pixel size. The data were taken in single shot mode in order to choose a fresh sample region after every shot. The background caused by the flare of the pump beam was subtracted from the delayed probe and reference peaks. The remaining intensity was integrated over the delayed spots and normalized with the integral over the corresponding spots of the unpumped shot. No data binning according to accelerator energy and pulse intensity was applied due to the small 


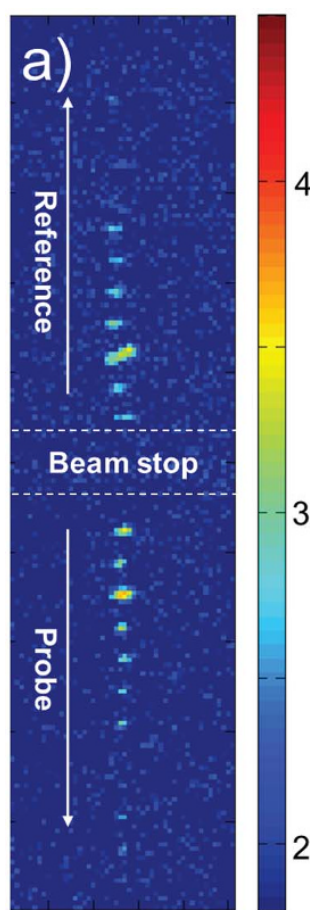

unpumped

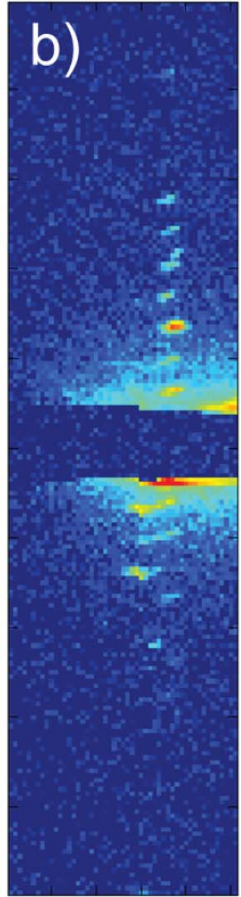

pumped \#1

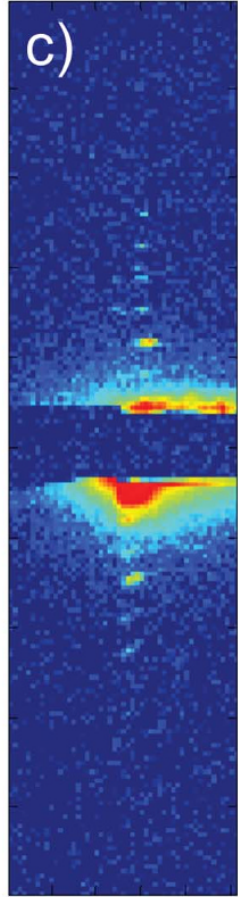

pumped \#2

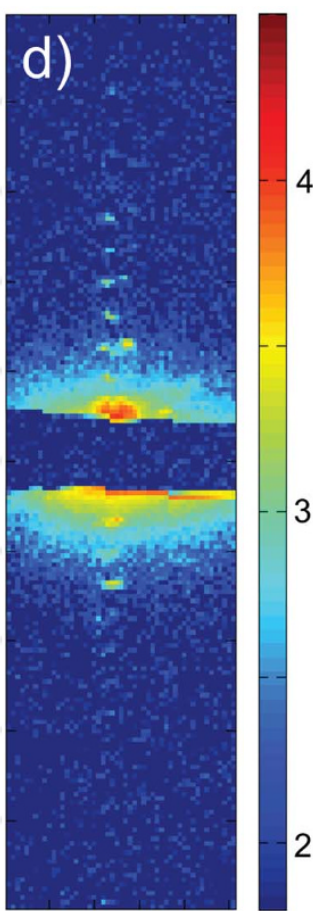

pumped \#3

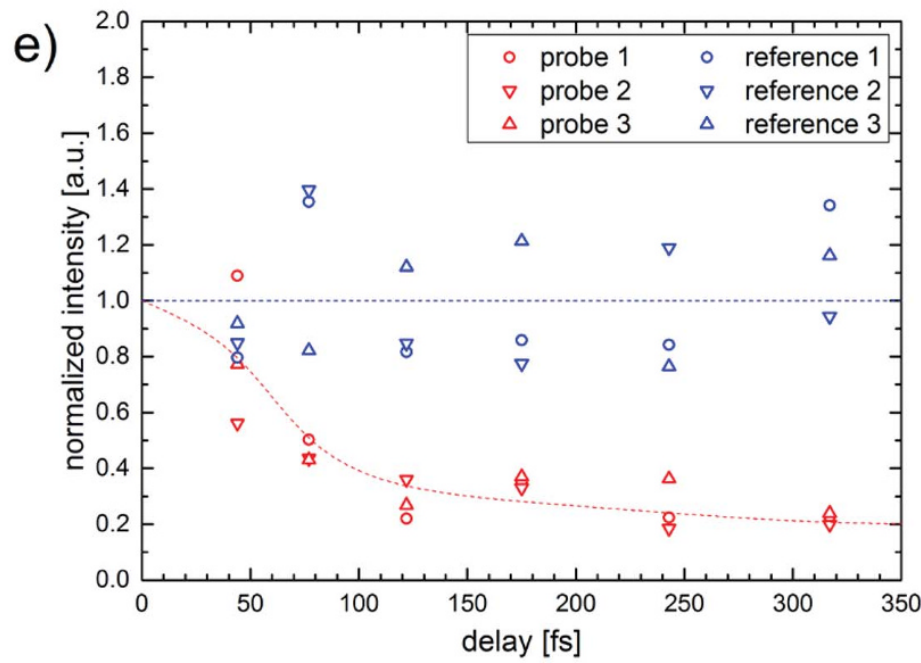

Figure 3 Single-shot time resolved X-ray diffraction measurements. The experimental geometry is the one depicted in Fig. 1, however using a 2dimensional pixel detector. The centre of the detector is protected by a beam stop. Panels (a)-(d) show the signal of the probe and reference beams on a logarithmic scale $(\log 10)$. Panel (a) was recorded with the pump beam blocked upstream of the sample, panels (b)-(d) display three single shot measurements at the full pump power density of $\approx 3 \times 10^{14} \mathrm{~W} / \mathrm{cm}^{2}$, meaning that no attenuators were used in the pump beam. Panel (e) shows the intensities integrated over the spots of $(\mathrm{b})-(\mathrm{d})$, normalized with the corresponding intensities of (a), versus delay time. The probe pulses decay due to the destruction of the Bi crystal lattice, whereas the reference signals remain constant. The dashed lines merely serve to guide the eye and do not represent experimental data.

number of single-shot experiments. This is the main cause for the fairly large scatter of the data points.

A route for improvement. Two main limitations of the described set-up are due to the grating performance: (i) The low efficiencies at high channel numbers needs to be enhanced by improving the grating efficiencies. For such weak gratings the efficiency scales with the square of the height of the grating lines. As the channel efficiency is determined by the efficiency product of both corresponding gratings, already a doubling of the structure height in each grating would lead to a channel efficiency increase by a factor of 16 . Such an increase in structure height could for example be achieved by using the gratings in a tilted geometry ${ }^{30}$. (ii) The strong shot-to-shot fluctuation of the relative peak heights can be explained by a fluctuating illumination of the gratings. As these devices have inhomogeneous efficiency distributions across their area, this will translate into fluctuations of the channel efficiencies. The correlation with the accelerator energy and thus with the photon energy is due to the corresponding changes in $S_{n}$ diffraction angle, causing the beam to illuminate a different part of - or even partly miss - the $R_{n}$ gratings. This problem must be addressed by fabricating larger and more homogeneous gratings.

1. Sokolowski-Tinten, K. et al. Femtosecond X-ray measurement of coherent lattice vibrations near the Lindemann stability limit. Nature 422, 287-289 (2003).

2. Elsaesser, T. \& Woerner, T. Photoinduced structural dynamics of polar solids studied by femtosecond X-ray diffraction. Acta Cryst. A66, 168-178 (2010).

3. Rundquist, A. et al. Phase-Matched Generation of Coherent Soft X-rays. Science 280, 1412-1415 (1998).

4. Schoenlein, R. W. et al. Generation of Femtosecond Pulses of Synchrotron Radiation. Science 287, 2237-2240 (2000). 
5. Ackermann, W. et al. Operation of a free-electron laser from the extreme ultraviolet to the water window. Nature Photon. 1, 336-341 (2007).

6. Emma, P. et al. First lasing and operation of an ångstrom-wavelength freeelectron laser. Nature Photon. 4, 641-647 (2010).

7. Ishikawa, T. et al. A compact X-ray free-electron laser emitting in the subångström region. Nature Photon. 6, 540-544 (2012).

8. Bressler, C. et al. Femtosecond XANES Study of the Light-Induced Spin Crossover Dynamics in an Iron(II) Complex. Science 323, 489-492 (2009).

9. Fritz, D. M. et al. Ultrafast bond softening in bismuth: Mapping a solid's interatomic potential with X-rays. Science 315, 633-636 (2007).

10. Johnson, S. L. et al. Nanoscale Depth-Resolved Coherent Femto-second Motion in Laser-Excited Bismuth. Phys. Rev. Lett. 100, 155501 (2008).

11. Vinko, S. M. et al. Creation and diagnosis of a solid-density plasma with an X-ray free-electron laser. Nature 482, 59-63 (2012).

12. Glownia, J. M. et al. Time-resolved pump-probe experiments at the LCLS. Opt. Express 18, 17620-17630 (2010).

13. Tavella, F. et al. Few-femtosecond timing at fourth-generation X-ray light sources. Nature Photon. 5, 162-165 (2011).

14. Löhl, F. et al. Electron bunch timing with femtosecond precision in a superconducting free-electron laser. Phys. Rev. Lett. 104, 144801 (2010).

15. Harmand, M. et al. Achieving few-femtosecond time-sorting at hard X-ray freeelectron lasers. Nature Phot. 7, 215-218 (2013).

16. Sorgenfrei, F. et al. The extreme ultraviolet split and femtosecond delay unit at the plane grating monochromator beamline PG2 at FLASH. Rev. Sci. Instrum. 81, 043107-7 (2010).

17. Castagna, J. C. et al. X-ray split and delay system for soft $\mathrm{x}$ rays at LCLS". I. of Physics: Conference Series 425, 152021-5 (2013).

18. Roseker, W. et al. Performance of a picosecond X-ray delay line unit at $8.39 \mathrm{keV}$. Opt. Lett. 34, 1768-1770 (2009).

19. Roseker, W. et al. Development of a hard X-ray delay line for X-ray photon correlation spectroscopy and jitter-free pump-probe experiments at X-ray freeelectron laser sources. J. Synchrotron Rad. 18, 481-491 (2011).

20. Villoresi, P. Compensation of optical path lengths in extreme-ultraviolet and softx-ray monochromators for ultrafast pulses. Applied Optics 38, 6040-6049 (1999).

21. Robert, A. et al. The X-ray Correlation Spectroscopy instrument at the Linac Coherent Light Source. J. of Physics: Conference Series 425, 212009 (2013).

22. Sciaini, G. et al. Electronic acceleration of atomic motions and disordering in bismuth. Nature 458, 56-60 (2009).

23. Chapman, H. N. et al. Femtosecond X-ray protein nanocrystallography. Nature 470, 73-77 (2011).

24. Barty, A. et al. Self-terminating diffraction gates femtosecond X-ray nanocrystallography measurements. Nature Photon. 6, 35-40 (2012)

25. David, C. et al. Nanofocusing of hard X-ray free electron laser pulses diamond based Fresnel zone plates. Sci. Rep. 1, 57 (2011).

26. Vila-Comamala, J. et al. Ultra-high resolution zone-doubled diffractive X-ray optics for the multi-keV regime. Opt. Express 19, 175-184 (2011).
27. Aaltonen, T. et al. Atomic layer deposition of iridium thin films. J. Electrochem. Soc. 151, G489-G492 (2004).

28. Mozzanica, A. et al. A single photon resolution integrating chip for microstrip detectors, Nuclear Instrum. Meth. Phys. Res. A 633, 29-32 (2011).

29. Herrmann, S. et al. CSPAD-140 $k$ : A versatile detector for LCLS experiments, Nuclear Instrum. Meth. Phys. Res. A 718, 550-553 (2011).

30. David, C. et al. A wavelength tunable diffractive transmission lens for hard $\mathrm{x}$-rays. Appl. Phys. Lett. 79, 1088-1090 (2001).

\section{Acknowledgments}

Portions of this research were carried out at the cSAXS beam line of the Swiss Light Source (SLS), Villigen, Switzerland, and the XCS instrument of the Linac Coherent Light Source (LCLS) at the SLAC National Accelerator Laboratory. LCLS is an Office of Science User Facility operated for the U.S. Department of Energy Office of Science by Stanford University. The authors thank the SLS and LCLS machine and experiments teams for their great support. The research leading to these results has received funding from the European Community's Seventh Framework Programme (FP7/2007-2013) under grant agreement no.290605 (PSI-FELLOW/COFUND).

\section{Author contributions}

The experiment was conceived by C.D. and P.K. The experimental hardware, including the diffraction gratings, was developed, fabricated, and characterised by C.D., P.K., M.S., I.M., G.C., S.H., A.M., A.D., E.F. and M.R. The measurements at LCLS were carried out by C.D., P.K., M.S., S.S., I.V., C.J.M., A.M., Y.K., G.C., S.H., D.M.F. and A.R. The LCLS data were analysed by P.K. with contributions from A.R. The manuscript was written by C.D. with extensive contributions from P.K. and suggestions from all other authors.

\section{Additional information}

Competing financial interests: The authors declare no competing financial interests.

How to cite this article: David, C. et al. Following the dynamics of matter with femtosecond precision using the X-ray streaking method. Sci. Rep. 5, 7644; DOI:10.1038/srep07644 (2015)

This work is licensed under a Creative Commons Attribution 4.0 International License. The images or other third party material in this article are included in the article's Creative Commons license, unless indicated otherwise in the credit line; if the material is not included under the Creative Commons license, users will need to obtain permission from the license holder in order to reproduce the material. To view a copy of this license, visit http://creativecommons.org/licenses/by/4.0/ 